\title{
Physical Nature of Critical Wave Functions in Fibonacci Systems
}

\author{
Enrique Maciá* and Francisco Domínguez-Adame \\ Departamento de Física de Materiales, Facultad de Físicas, Universidad Complutense, E-28040 Madrid, Spain
}

(Received 28 April 1995; revised manuscript received 12 December 1995)

\begin{abstract}
We report on a new class of critical states in the energy spectrum of general Fibonacci systems. By introducing a transfer matrix renormalization technique, we prove that the charge distribution of these states spreads over the whole system, showing transport properties characteristic of electronic extended states. Our analytical method is a first step to find out the link between the spatial structure of critical wave functions and their related transport properties.
\end{abstract}

PACS numbers: 71.23.Ft, 61.44.-n

The notion of critical wave function (CWF) has evolved continuously since its introduction in the study of aperiodic systems [1], leading to a somewhat confusing situation. For instance, references to self-similar, chaotic, quasiperiodic, latticelike, or quasilocalized CWFs can be found in the literature depending on the different criteria adopted to characterize them [2-6]. Generally speaking, CWFs exhibit a rather involved oscillatory behavior, displaying strong spatial fluctuations which show distinctive self-similar features in some instances. As a consequence, the notion of an envelope function, which has been most fruitful in the study of both extended and localized states, is mathematically ill-defined in the case of CWFs, and other approaches are required to properly describe them and to understand their structure.

Most interestingly, the possible existence of extended states in several kinds of aperiodic systems, including both quasiperiodic [7-10] and nonquasiperiodic ones $[4,11]$, has been discussed in the last few years spurring the interest on the precise nature of CWFs and their role in the physics of aperiodic systems. From a rigorous mathematical point of view the nature of a state is uniquely determined by the measure of the spectrum to which it belongs. In this way, since it has been proven that Fibonacci lattices have purely singular continuous energy spectra [12], we must conclude that the associated electronic states cannot be, strictly speaking, extended in the Bloch's sense. This result holds for other aperiodic lattices (Thue-Morse, period doubling) as well [13], and it may be a general property of the spectra of self-similar aperiodic systems [14]. On the other side, from a physical viewpoint, the states can be classified according to their transport properties which, in turn, are determined by the spatial distribution of the wave function amplitudes (charge distribution). Thus, conducting, crystalline systems are described by periodic Bloch states, whereas insulating systems are described by exponentially decaying wave functions corresponding to localized states. In this sense, since the amplitudes of CWFs in a Fibonacci lattice do not tend to zero at infinity but are bounded below throughout the system [15], one may expect their physical behavior to be more similar to that corresponding to extended states than to localized ones.
In this Letter we are going to show analytically that a subset of the CWFs belonging to general Fibonacci systems are extended from a physical point of view. This result widens the notion of extended wave function to include electronic states which are not Bloch functions, and it is a relevant first step to clarify the precise manner in which the quasiperiodic order of Fibonacci systems influences their transport properties [16]. To this end we present, in the first place, a new renormalization approach opening, in a natural way, an algebraic formalism which allows us to give a detailed analytical account of the transport properties of CWFs for certain particular values of the energy. In the second place, we study the relationship between the spatial structure of CWFs and their transport properties, showing that self-similar wave functions are those exhibiting higher transmission coefficients in finite Fibonacci systems.

The formalism we are going to introduce is based on the transfer matrix technique, where the solution of the Schrödinger equation is obtained by means of a product of $2 \times 2$ matrices. Real-space renormalization group approaches, based on decimation schemes, have proved themselves very successful in order to numerically obtain the energy spectrum of deterministic aperiodic systems $[17,18]$. The convenience for such procedures stems from the fact that, by construction, a given transfer matrix relates only three consecutive sites along the lattice, so that by decimating the original chain into successively longer blocks we are able to describe the electronic state corresponding to sites farther and farther apart. In this context, the key point of our procedure consists of the fact that we renormalize the set of transfer matrices instead of the lattice itself. Since these matrices contain all the relevant information concerning the dynamics of the electrons, our approach becomes especially well suited to describe the characteristic features associated with the long-range order of the underlying Fibonacci system for, as we will see below, it preserves the original quasiperiodic order of the lattice at any stage of the renormalization process.

Let us start by considering a general Fibonacci system in which both diagonal and off-diagonal terms are present 
in the Hamiltonian $[18,19]$

$$
\begin{aligned}
H=\sum_{n} & \left\{V_{n}|n\rangle\left\langle n\left|+t_{n, n+1}\right| n\right\rangle\langle n+1|\right. \\
& \left.+t_{n, n-1}|n\rangle\langle n-1|\right\},
\end{aligned}
$$

where $V_{n}$ is the on-site energy and $t_{n, n \pm 1}$ are the nearestneighbor hopping integrals. This Hamiltonian can be cast in terms of the following matrices:

$$
\begin{aligned}
X \equiv\left(\begin{array}{cc}
\frac{E-\beta}{t_{A B}} & -1 \\
1 & 0
\end{array}\right), & Y \equiv\left(\begin{array}{cc}
\gamma^{-1} \frac{E-\alpha}{t_{A B}} & -\gamma^{-1} \\
1 & 0
\end{array}\right), \\
Z \equiv\left(\begin{array}{cc}
\frac{E-\alpha}{t_{A B}} & -\gamma \\
1 & 0
\end{array}\right), & W \equiv\left(\begin{array}{cc}
\frac{E-\alpha}{t_{A B}} & -1 \\
1 & 0
\end{array}\right),
\end{aligned}
$$

where $E$ is the electron energy, $\alpha(\beta)$ denote the onsite energies of sites $A(B), t_{A B}=t_{B A}$ and $t_{A A}$ are the corresponding hopping integrals, and $\gamma \equiv t_{A A} / t_{A B}>$ 0 . Making use of these matrices, and imposing cyclic boundary conditions, we can translate the atomic sequence $A B A A B \ldots$ describing the topological order of the Fibonacci lattice to the transfer matrix sequence ...XZYXZYXWXZYXW describing the behavior of electrons moving through it. In spite of its greater apparent complexity, we realize that by renormalizing this transfer matrix sequence according to the blocking scheme $R_{A} \equiv Z Y X$ and $R_{B} \equiv W X$, we get the considerably simplified sequence $\ldots R_{B} R_{A} R_{A} R_{B} R_{A}$. The subscripts in the Rs matrices are introduced to emphasize the fact that the renormalized transfer matrix sequence is also arranged according to the Fibonacci sequence and, consequently, the topological order present in the original lattice is preserved by the renormalization process. Let $N=F_{n}$ be the number of lattice sites, where $F_{n}$ is a Fibonacci number obtained from the recursive law $F_{n}=F_{n-1}+F_{n-2}$, with $F_{1}=1$ and $F_{0}=1$. It can then be shown by induction that the renormalized sequence contains $n_{A} \equiv F_{n-3}$ matrices $R_{A}$ and $n_{B} \equiv F_{n-4}$ matrices $R_{B}$.

We will now use two properties of the Rs matrices to develop our procedure. First, they are unimodular (i.e., their determinant equals unity) for any choice of the system parameters and for any value of the electron energy. Second, they commute for certain values of the energy. In fact, after some algebra we get

$$
\left[R_{A}, R_{B}\right]=\frac{\alpha\left(1+\gamma^{2}\right)-E\left(1-\gamma^{2}\right)}{\gamma}\left(\begin{array}{cc}
1 & 0 \\
E+\alpha & -1
\end{array}\right),
$$

where we have defined the origin of energies in such a way that $\beta=-\alpha$ and $t_{A B} \equiv 1$. This commutator is considerably simplified for the two cases mostly discussed in the literature, namely, the on-site $(\gamma \equiv 1)$ and transfer $(\alpha \equiv 0)$ models. The expression (2) shows that the on-site model is intrinsically noncommutative, for the commutator vanishes only in the trivial periodic case. On the contrary, in the transfer model the $R$ matrices commute for the energy value $E=0$, which corresponds to the center of the energy spectrum. Most interestingly, according to expression (2), there exists always one energy satisfying the relation

$$
E=\alpha \frac{1+\gamma^{2}}{1-\gamma^{2}},
$$

for any realization of the mixed model (i.e., for any combination of $\alpha$ and $\gamma \neq 1$ values). For these energies the condition $\left[R_{A}, R_{B}\right]=0$ is fulfilled and, making use of the Cayley-Hamilton theorem for unimodular matrices [20], the global transfer matrix of the system, $M(N) \equiv R_{A}^{n_{A}} R_{B}^{n_{B}}$, can be explicitly evaluated in terms of Chebyshev polynomials of the second kind. Alternatively, the required power matrices can be evaluated by diagonalizing them to a common basis. From the knowledge of $M(N)$ the condition for the considered energy value to be in the spectrum, $|\operatorname{Tr}[M(N)]| \leq 2$, can be readily checked and, afterwards, relevant magnitudes describing their transport properties can be determined explicitly. In this way, given any arbitrary Fibonacci lattice, we are able to obtain a subset of its energy spectrum whose eigenstates can be studied analytically.

We look for energies where the corresponding wave functions do not grow exponentially with the system size. This leads to the condition that the modulus of the common eigenvalues of matrices $R_{A}$ and $R_{B}$ should be unity. This condition is fulfilled by the subset of energies (3) satisfying $\left|2 \gamma-\sqrt{\alpha^{2}+4}\right| \leq|\alpha|$. The global transfer matrices corresponding to these energies can be expressed, after lengthy algebra, in the closed form

$$
M(N)=\frac{1}{\sin \phi}\left(\begin{array}{cc}
\sin [(N+1) \phi] & -\gamma \sin (N \phi) \\
\gamma^{-1} \sin (N \phi) & -\sin [(N-1) \phi]
\end{array}\right),
$$

where $2 \cos \phi \equiv \sqrt{E^{2}-\alpha^{2}}$. From expression (4) we get $\operatorname{Tr}[M(N)]=2 \cos (N \phi)$ and, consequently, we can ensure that these energies belong to the spectrum in the quasiperiodic limit $(N \rightarrow \infty)$. Now, we proceed to the calculation of the transmission coefficient, $\tau(N)$, a magnitude directly related to the Landauer resistivity [21], $\rho$, by embedding the Fibonacci lattice in an infinite periodic arrangement of identical atoms connected by hopping integrals $t \equiv 1$. In this way we obtain

$$
\begin{aligned}
\tau(N) & =\frac{1}{1+\rho} \\
& =\frac{1}{1+\left[\left(1-\gamma^{2}\right)^{2} /\left(4-E^{2}\right) \gamma^{2}\right] \sin ^{2}(N \phi)} .
\end{aligned}
$$

Two important conclusions can be drawn from this expression. In the first place, the transmission coefficient is always bounded below for any lattice length, which proves the true extended character of the related states. In the second place, since the factor multiplying the sine in the denominator of expression (5) only vanishes in the case $\gamma=1$, the critical states we are considering do not 
verify, in general, the transparency condition $\tau=1$ in the quasiperiodic limit. However, it is possible to find states satisfying the transparency condition in finite Fibonacci systems whose length satisfies the relationship $N \phi=k \pi$, $k=1,2 \ldots$, which, in turn, implies

$$
E(k)= \pm \sqrt{\alpha^{2}+4 \cos ^{2}(k \pi / N)},
$$

with $|\alpha|<2$. In this way, the transparent states can be classified according to a well defined scheme determined by the integer $k$.

Now let us consider the spatial structure of the states corresponding to expressions (3) and (6). Representative examples are, respectively, shown in Figs. 1 and 2. The charge distribution shown in Fig. 1(a) corresponds to the state of energy $E_{1}=-1.25$ in a Fibonacci chain with $N=F_{16}=1597$ sites and lattice parameters $\gamma=2$ and $\alpha=0.75$. The overall behavior of the wave function amplitudes, which we have calculated exactly with the aid of our matrix formalism making use of the initial conditions $\varphi_{0}=0$ and $\varphi_{1}=1$, clearly indicates its extended character. At this point it is worth mentioning that, albeit its ap-
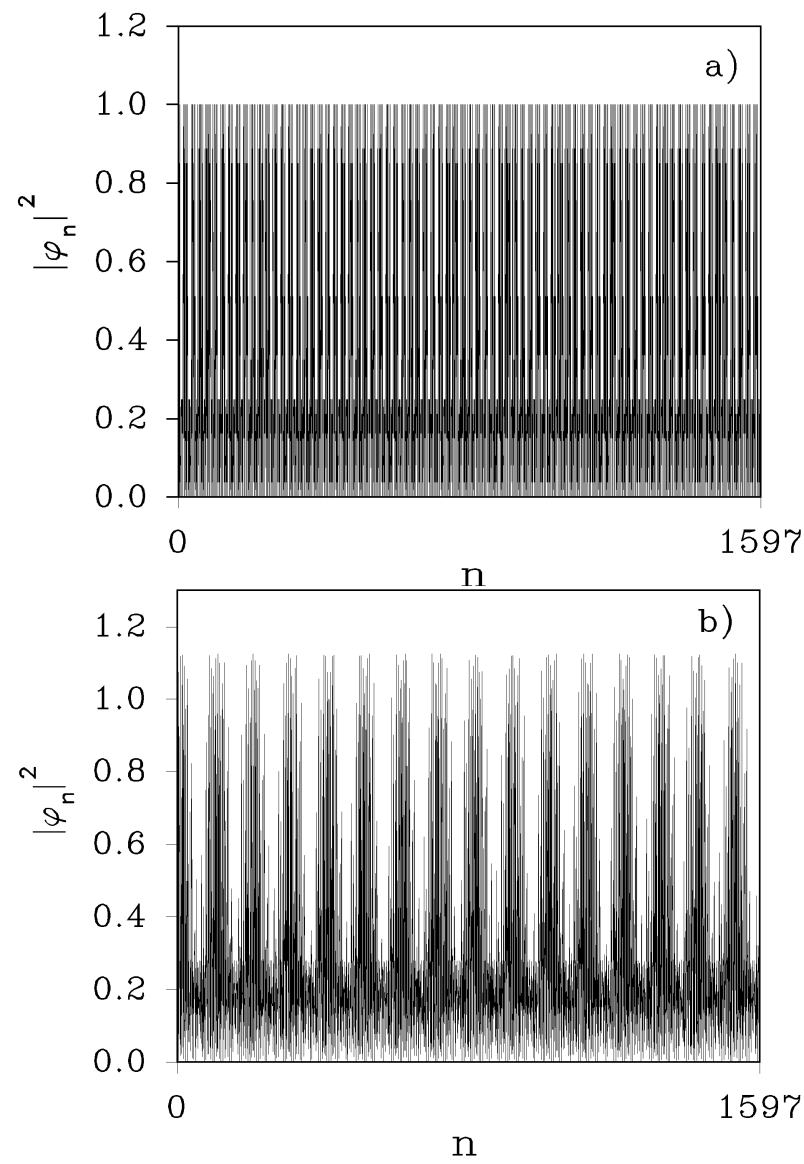

FIG. 1. Electronic charge distribution in Fibonacci lattices with $N=F_{16}$ and (a) $\gamma=2, \alpha=0.75, E_{1}=-1.25$ and (b) $\gamma=2, \alpha=0.5, E_{2}=-5 / 6$. Their transmission coefficients are, respectively, $\tau\left(E_{1}\right)=0.5909 \ldots$ and $\tau\left(E_{2}\right)=0.7425 \ldots$ pearance, this wave function is nonperiodic: The sequence of values taken by the wave function amplitude is arranged according to a quasiperiodic sequence. Figure 1(b) shows the charge distribution corresponding to the state of energy $E_{2}=-5 / 6$ in a system of the same length and model parameters $\gamma=2.0$ and $\alpha=0.5$. At first sight, by comparing both figures, one may be tempted to think that the transmission coefficient corresponding to the wave function plotted in Fig. 1(a) must be higher than that corresponding to the wave function shown in Fig. 1(b), because the charge distribution of the former along the system is more homogeneous than that corresponding to the latter. Actually, however, making use of expression (5), we found $\tau\left(E_{1}\right)=0.5909 \ldots$ and $\tau\left(E_{2}\right)=0.7425 \ldots$, which is precisely the opposite case.

To gain further insight into the behavior of the wave function at all length scales we have performed a multifractal analysis of these states. The amplitude distribution of the electronic states has been characterized by the scaling of moments $\mu_{q}(N)$ of order $q$, associated with their charge distribution, with the system size (for a definition of those moments see, e.g., Ref. [22]). The multifractal dimension $D_{q}$ is determined via the scaling $\mu_{q}(N) \sim N^{(1-q) D_{q}}$ for $q \neq 0$. In all cases studied we have found that $D_{q}=1$, for all $q$, and for system sizes as large as $N=F_{30}=1346269$. Thus the lack of multifractality along with the fact that $D_{q}$ equals the spatial dimension clearly confirms that these states uniformly spread over the whole system.

In Fig. 2 we show the typical charge distribution corresponding to states given by expression (6) for a system with $N=F_{17}=2584$ and model parameters $\gamma=2$ and $\alpha=0.1$. These states exhibit, in general, a characteristic self-similar structure in a statistical sense. Making use of (5) we get $\tau[E(k)]=1$, indicating that, in finite Fibonacci

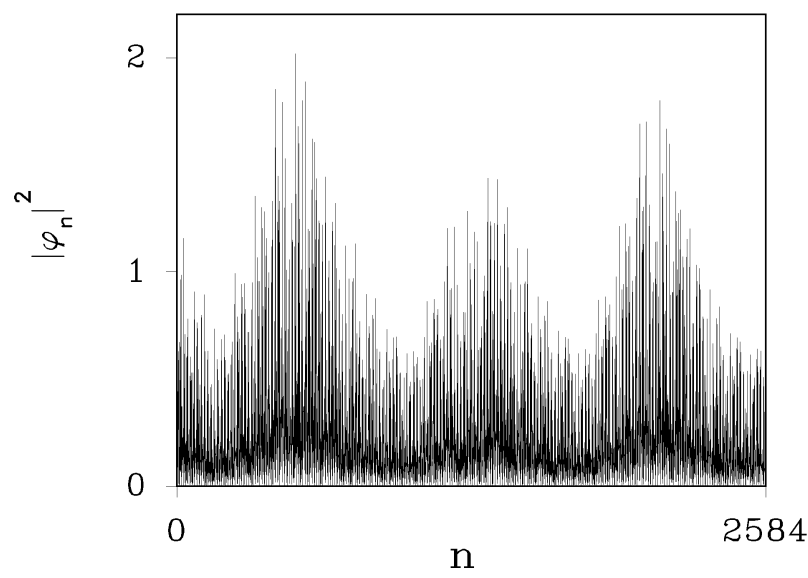

FIG. 2. Electronic charge distribution in a Fibonacci lattice with $N=F_{17}, \quad \gamma=2, \quad \alpha=0.1$, and $E(k=1160)=$ $-\sqrt{\alpha^{2}+4 \cos ^{2}(1160 \pi / N)}$ corresponding to a transparent state for which $\tau(E)=1$. Statistical self-similar features are clearly seen. 
systems, this kind of self-similar states exhibit better transport properties than those corresponding to more homogeneous wave functions shown in Fig. 1.

Summing up, in this paper we show that, for general Fibonacci systems in which both diagonal and off-diagonal quasiperiodic order is present in their model Hamiltonian, there exists a set of extended critical states for which $\tau \neq 0$ when $N \rightarrow \infty$. At the same time, these energies do not correspond to transparent states in the quasiperiodic limit $(\tau<1)$. Therefore, the transmission coefficient for this class of critical states satisfies $0<\tau<1$, in agreement with the view of critical states as intermediate between periodic Bloch wave functions $(\tau=1)$ and Anderson localized states $(\tau=0)$. On the other side, we also show that it is possible to design finite Fibonacci systems able to support transparent states for certain specific energies, and that the charge distribution of such states in the space display self-similar patterns.

This we have shown by means of a transfer matrix renormalization technique which allows us to unveil the effects of short-range correlations by grouping $A B A$ sites and $A B$ sites into the matrices $R_{A}$ and $R_{B}$, respectively. In this sense we can properly ensure that these states are characteristic of the quasiperiodic order of the underlying lattice. Interestingly we note that similar results concerning extended states in Thue-Morse chains have been recently reported in the literature [23]. We wish to stress that the algebraic approach presented in this work can be extended in a straightforward manner to other kinds of aperiodic systems based on substitution sequences, and therefore it can be relevant in order to attain a unified treatment of physical properties of aperiodic systems.

We are greatly indebted to Roland Ketzmerick for very useful discussions and detailed calculations as well as for his good manners in science. It is with great pleasure that we thank Angel Sánchez for illuminating conversations. We also thank Victoria Hernández for interesting comments. This work is supported by CICYT under Project No. MAT95-0325.

*Also at Instituto de Estudios Interdisciplinares, El Guijo, Z4 Galapagar, E-28260 Madrid, Spain. Electronic address: fimat04@emducms1.sis.ucm.es

†Electronic address: adame@eucmos.sim.ucm.es

[1] S. Ostlund and R. Pandit, Phys. Rev. B 29, 1394 (1984).
[2] M. Kohmoto and J.R. Banavar, Phys. Rev. B 34, 563 (1986).

[3] M. Kohmoto, B. Sutherland, and C. Tang, Phys. Rev. B 35, 1020 (1987).

[4] M. Severin, M. Dulea, and R. Riklund, J. Phys. Condens. Matter 1, 8851 (1989).

[5] C. S. Ryu, G. Y. Oh, and M. H. Lee, Phys. Rev. B 46, 5162 (1992).

[6] W. Gellermann, M. Kohmoto, B. Sutherland, and P.C. Taylor, Phys. Rev. Lett. 72, 633 (1994).

[7] V. Kumar and G. Ananthakrishna, Phys. Rev. Lett. 59, 1476 (1987).

[8] X. C. Xie and S. Das Sarma, Phys. Rev. Lett. 60, 1585 (1988); G. Ananthakrishna and V. Kumar, Phys. Rev. Lett. 60, 1586 (1988).

[9] V. Kumar, J. Phys. Condens. Matter 2, 1349 (1990).

[10] A. Chakrabarti, S. N. Karmakar, and R. K. Moitra, Phys. Lett. A 168, 301 (1992); G. Y. Oh, C.S. Ryu, and M. H. Lee, J. Phys. Condens. Matter 4, 8187 (1992); A. Chakrabarti, S. N. Karmakar, and R. K. Moitra, Phys. Rev. B 50, 13276 (1994).

[11] S. Sil, S. N. Karmakar, R. K. Moitra, and A. Chakrabarti, Phys. Rev. B 48, 4192 (1993).

[12] A. Süto, J. Stat. Phys. 56, 525 (1989); J. Bellisard, B. Iochum, E. Scoppola, and D. Testard, Commun. Math. Phys. 125, 527 (1989).

[13] J. Bellisard, A. Bovier, and J.-M. Ghez, Commun. Math. Phys. 135, 379 (1991); A. Bovier and J.-M. Ghez, Commun. Math. Phys. 158, 45 (1993).

[14] A. Bovier and J. M. Ghez, J. Phys. A 28, 2313 (1995).

[15] B. Iochum and D. Testard, J. Stat. Phys. 65, 715 (1991).

[16] E. Maciá and F. Domínguez-Adame, Phys. Rev. B 50, 16856 (1994); F. Domínguez-Adame, E. Maciá, and A. Sánchez, Phys. Rev. B 51, 878 (1995).

[17] Q. Niu and F. Nori, Phys. Rev. Lett. 57, 2057 (1986); Q. Niu and F. Nori, Phys. Rev. B 42, 10329 (1990); F. Piéchon, M. Benakli, and A. Jagannathan, Phys. Rev. Lett. 74, 5248 (1995).

[18] E. Maciá, F. Domínguez-Adame, and A. Sánchez, Phys. Rev. E 50, 679 (1994), and references therein.

[19] C. Sire and R. Mosseri, J. Phys. (Paris) 51, 1569 (1990).

[20] See, for example, J.M. Luck, in Fundamental Problems in Statisitical Mechanics VIII (Elsevier, New York, 1994).

[21] R. Landauer, IBM J. Res. Dev. 1, 223 (1957).

[22] T. C. Halsey, M. H. Jensen, L. P. Kadanoff, I. Procaccia, and B.I. Shraiman, Phys. Rev. A 33, 1141 (1986); A. Sánchez, E. Maciá, and F. Domínguez-Adame, Phys. Rev. B 49, 147 (1994).

[23] A. Chakrabarti, S. N. Karmakar, and R. K. Moitra, Phys. Rev. Lett. 74, 1403 (1995). 